chloride and sodium hydroxide and/or caused a decomposition of the reaction product, the $\mathrm{SPO}_{3}{ }^{3-}$ ion.

A closer examination of this phenomenon has now revealed that silicone grease is a catalyst of the hydrolysis of the phosphorothioate ion in strongly alkaline solution.

At $50^{\circ}$ and in $1 \mathrm{M}$ sodium hydroxide the rate of hydrolysis constant of the phosphorothioate ion was found to be $2.5 \times 10^{-4} \mathrm{~min}^{-1}$, as measured in a carefully cleaned glass flask. In the presence of $30 \mathrm{mg} / 100 \mathrm{ml}$. of Dow Corning high vacuum silicone grease the rate of hydrolysis constant increased to $2 \cdot 1 \times 10^{-3} \mathrm{~min}^{-1}$, that is, nearly nine times the value obtained with a clean flask. The rates of hydrolysis were followed by determination of liberated orthophosphate according to Gomori ${ }^{2}$.

It was further noticed that a strongly alkaline medium is necessary for silicone grease catalysed hydrolysis of the phosphorothioate ion. No catalysis is observed, for example, at $p \mathbf{H} 8 \cdot 0$. This is probably due to the fact that in strongly alkaline medium silicone grease doos no longer adhere to the walls of the glass flask, but is emulsified in the liquid, thus incroasing the catalytic surface tremendously.

Mechanistically the hydrolysis of the phosphorothioate ion in alkaline medium is likely to be due to a water attack on that ion $^{3}$. The catalytic action of silicone grease may therefore involve activation of water molecules hydrogen bonded to the surface of the silicone grease. A similar water activation, caused by negatively charged oxygen atoms, is observed, for example, in the hydrolysis of certain S-substituted phosphorothioic acids ${ }^{4}$. STIG ÁkerfeLd

Research Institute of National Defence, Department 1, Sundbyberg 4, Sweden.

1 Akerfeldt, S., Acta Chem. Scand., 14, 1980 (1960).

${ }^{2}$ Gormori, G., J. Lab. Clin. Med., 27, 955 (1942).

${ }^{3}$ Ramsay, O. B., Univ. Microfilms No. 60-3607.

- Ákerfeldt, S., Acta Chem. Scand., 17, 319 (1963).

\section{Identification of Organic Compounds from Food Odours by Pyrolysis}

IN preparing the combined technique of pyrolysis and gas chromatography for the general purpose of identification of compounds in food odours we now have carried out pyrolysis experiments using some aliphatic compounds as test substances. In a previous note I reported the detection of the benzene ring in organic compounds by pyrolysis and gas chromatography ${ }^{1}$, using a Pye apparatus from which the argon detector was removed and replaced by a flame ionization detector following the method of Murray and Williams ${ }^{2}$. A column carrying 30 per cent 'Apiezon $L$ ' on 'Chromosorb $W$ ' was used. The quartz pyrolysis tube (length, $20 \mathrm{~cm}$; inner diam., $2 \mathrm{~mm}$ ) was filled with 'Chromosorb $P$ ' over a length of $10 \mathrm{~cm}$ and was connected to the top of the column. Nitrogen was used as the carrier gas at a flow of $32 \mathrm{ml} . / \mathrm{min}$.

$50-5,000 \mu \mathrm{g}$ of the compound in the vapour form and in the presence of a large excess of air was injected through the pyrolysis tube, which was heated to $600^{\circ} \mathrm{C}$ by an electric furnace.

The peaks of the pyrolysis products were characterized using the Kovats index scale, because this index has been shown to be nearly independent of experimental conditions ${ }^{3}$.

The gas chromatograph thus was used as a "poor man's mass spectrometer", as first suggested by Keulemans".

Fig. 1 illustrates the results obtained in the pyrolysis of some $\mathrm{C}_{5}$ straight chain compounds with one functional group.

It is seen that every compound shows a characteristic pyrolysis pattern. The reproducibility of the pyrolysis patterns, obtained on repeated injections of the same compound, is good and eliminates the danger of misinterpretation of the results for identification purposes.

For a tentative interpretation of these "pyrograms"s use was made of the relation between boiling point and Kovats index as reported elsewhere ${ }^{3}$.
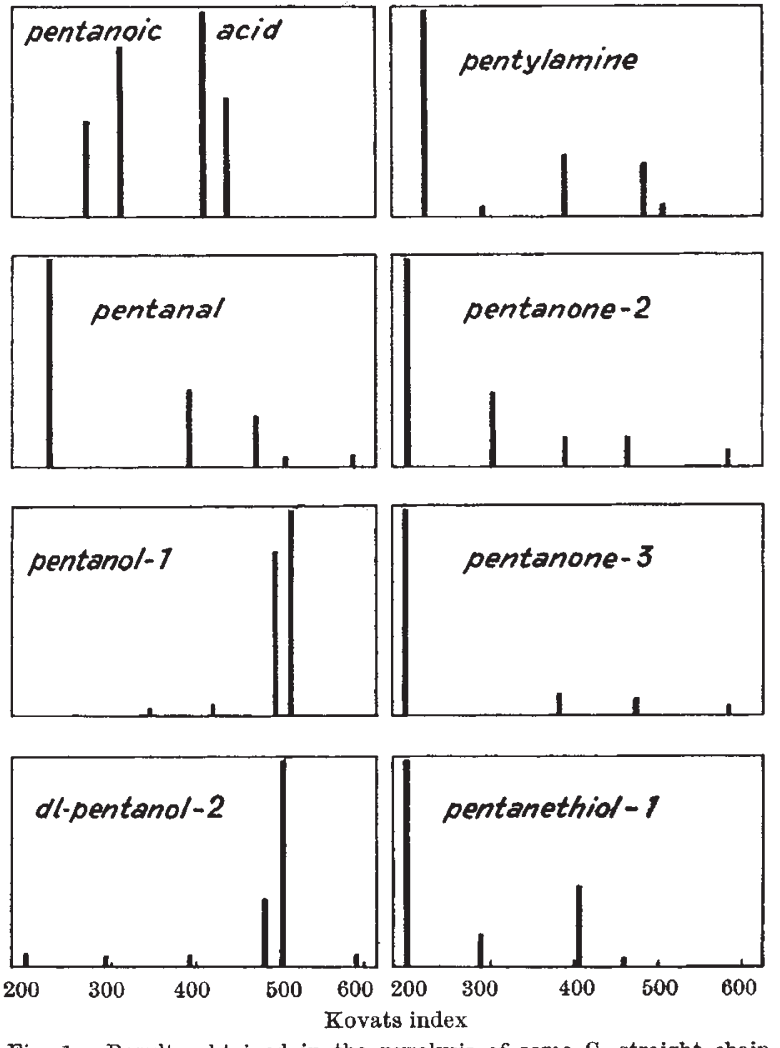

Fig. 1. Results obtained in the pyrolysis of some $\mathbf{C}_{5}$ straight chain compounds. Abscissa, Kovats index units. Ordinate, relative peak area as percentage of main peak

The main reaction during the pyrolysis of the alcohol seems to be removal of water from the molecule with the formation of the corresponding olefine. The pyrolysis of the acid results in the formation of a $\mathrm{C}_{4}$-hydrocarbon by decarboxylation (main peak at 404 index units).

The carbon chain in the amine, aldehyde and the ketone is completely disintegrated into smaller fragments.

Central Institute for Nutrition and

J. H. DHont

Food Research T.N.O., Utrecht.

${ }^{1}$ Dhont, J. H., Nature, 192, 747 (1961).

2 Murray, W. J., and Williams, A. F., Analyst, 86, 849 (1961).

s Dhont, J. H., Nature, 198, 990 (1963).

- Keulemans, A. I. M., in Gaschromatography : a Symposium . . ., Aug. 1957, 237 (New York, Academic Press, 1058).

${ }^{5}$ Parris, W. H., and Holland, P. D., British Plastics, 1 (1960).

\section{Effect of Reaction of Urea with Jute on the Photochemical Degradation of the Fibre}

Is the course of investigations carried out with the view of improving the resistance of jute fibre towards light by chemical modification, it was observod that a remarkablo protection against dogradation of the fibre by light can be achieved by reacting the fibre with urea in presence of a catalyst at an olevated temperature.

Reaction of urea with cotton ${ }^{1}$ has beon shown to have no appreciable effect on the usual textile properties of the fibre. In jute, however, marked changos in both physical and chemical proportios of the fibre are noticed, particularly in the susceptibility of the fibre towards photochemical degradation. Jute undergoes degradation under the influonce of light at a much higher rate than other vegetable fibres ${ }^{2}$ leading to loss in tensile strength, increase in alkali solubility ard fibro acidity, and a number of other changes in fibre properties ${ }^{3,4}$. Treatment with urea arrests these photochemical changes and this is illustrated by a sot of experimonts doscribod here with results shown in Tablo 1. 